\title{
Neutrophil extracellular trap cell death requires both autophagy and superoxide generation
}

Quinten Remijsen ${ }^{1,2,3}$, Tom Vanden Berghe ${ }^{2,3}$, Ellen Wirawan ${ }^{2,3}$, Bob Asselbergh ${ }^{4}$, Eef Parthoens ${ }^{4,5}$, Riet De Rycke ${ }^{4}$, Sam Noppen ${ }^{4}$, Michel Delforge ${ }^{6}$, Jean Willems ${ }^{1, *}$, Peter Vandenabeele ${ }^{2,3, *}$

${ }^{I} I R C$ - Laboratory of Biochemistry, Department of Medicine, KU Leuven Campus Kortrijk, Kortrijk, Belgium, ${ }^{2}$ Molecular Signaling and Cell Death Unit, Department for Molecular Biomedical Research, VIB, Ghent, Belgium; ${ }^{3}$ Molecular Signaling and Cell Death Unit, Department of Biomedical Molecular Biology, Ghent University, Ghent, Belgium; ${ }^{4}$ Microscopy Core Facility, Department for Molecular Biomedical Research, VIB, Ghent, Belgium; ${ }^{5}$ Department of Biomedical Molecular Biology, Ghent University, Ghent, Belgium; ${ }^{6}$ University Hospital Gasthuisberg, Department of Hematology, Leuven, Belgium

Neutrophil extracellular traps (NETs) are extracellular chromatin structures that can trap and degrade microbes. They arise from neutrophils that have activated a cell death program called NET cell death, or NETosis. Activation of NETosis has been shown to involve NADPH oxidase activity, disintegration of the nuclear envelope and most granule membranes, decondensation of nuclear chromatin and formation of NETs. We report that in phorbol myristate acetate (PMA)-stimulated neutrophils, intracellular chromatin decondensation and NET formation follow autophagy and superoxide production, both of which are required to mediate PMA-induced NETosis and occur independently of each other. Neutrophils from patients with chronic granulomatous disease, which lack NADPH oxidase activity, still exhibit PMA-induced autophagy. Conversely, PMA-induced NADPH oxidase activity is not affected by pharmacological inhibition of autophagy. Interestingly, inhibition of either autophagy or NADPH oxidase prevents intracellular chromatin decondensation, which is essential for NETosis and NET formation, and results in cell death characterized by hallmarks of apoptosis. These results indicate that apoptosis might function as a backup program for NETosis when autophagy or NADPH oxidase activity is prevented.

Keywords: neutrophil extracellular trap; granulocyte; chronic granulomatous disease; superoxide; autophagy; live cell imaging

Cell Research (2011) 21: 290-304. doi:10.1038/cr.2010.150; published online 9 November 2010

\section{Introduction}

Neutrophils are short lived but nevertheless very abundant phagocytic leukocytes that form a vital first line of defense against invading pathogens. Neutrophil contents are potentially harmful to the host and the release and activation of their microbicidal arsenal is strongly controlled by strict regulation of degranulation and superox-

\footnotetext{
*These two authors share equally senior authorship to this work. Correspondence: Peter Vandenabeele ${ }^{\mathrm{a}}$, Jean Willems ${ }^{\mathrm{b}}$

${ }^{a}$ Tel: +32-0-9-33-13760, Fax: +32-0-9-33-13609

E-mail: Peter.Vandenabeele@dmbr.vib-UGent.be

${ }^{\mathrm{b}}$ Tel: +32-56-246225, Fax: +32-56-246997

E-mail: Jean.Willems@kuleuven-kortrijk.be

Received 24 June 2010; revised 28 July 2010; accepted 1 September 2010; published online 9 November 2010
}

ide production, as well as by regulation of their life span and recruitment into tissues. In the absence of immunological challenge, differentiated neutrophils are committed to undergo caspase-dependent apoptosis within 24 to $48 \mathrm{~h}$ after their emigration from the bone marrow [1]. During infection, the rate of programmed cell death can be further modulated by various exogenous and endogenous stimuli that either extend or shorten the neutrophil life span [2].

In 2004, a novel type of cell death was reported, neutrophil extracellular trap (NET) cell death [3], which is also named NETosis [4]. Activation of NETosis has been shown to involve NADPH oxidase (Nox2)-mediated oxidative burst [5] and disintegration of the nuclear envelope and most granule membranes, which together result in massive vacuolization [6], intracellular decondensation of nuclear chromatin [7] and eventually formation of 
NETs [3]. An essential role in the regulation of NETosis is ascribed to phagocyte Nox2, a highly regulated membrane-associated multiprotein complex producing large amounts of superoxide that lead to an oxidative burst [5]. Phorbol myristate acetate (PMA) induces NETosis, but this is prevented by inhibition of NADPH oxidase activity (by diphenylene iodonium, DPI, [6]) and by its absence (as in patients with chronic granulomatous disease (CGD), caused by a congenital defect in Nox2 subunits [8]). Also typical during NETosis is the generation of many vesicles before plasma membrane rupture [6]. These vesicles have a double phospholipid bilayer and are believed to originate from the nuclear envelope $[6,9]$, which disintegrates during NET cell death. Finally, but still before plasma membrane permeabilization, nuclear chromatin decondenses and mixes with the contents of the granules; this is essential for formation of functional NETs $[7,10]$. Permeabilization of the neutrophil plasma membrane releases these chromatin structures, which are loaded with concentrated antimicrobial molecules, such as lactoferrin, BPI, LL-37 and histones [9, 11]. These structures can trap and possibly even kill microorganisms. Consequently, neutrophils can exert an antimicrobial effect beyond their life span [9].

Data collected over the past 5 years demonstrate the in vivo occurrence of NETosis in different clinical settings such as appendicitis [3], necrotizing fasciitis [12], pneumonia [13], sepsis [14], leishmaniasis [15] and small vessel vasculitis (SVV) [16], suggesting a pathophysiological relevance in these conditions. Recently, the kinetics of in vivo NET formation in murine lungs in response to Aspergillus infection was monitored [17]. The results showed that NETs are formed during the early stages of infection. In addition to the reported induction of NETosis formation by bacteria [3, 6, 12, 13], fungi [17-19] and protozoa [15], NETosis has also been shown to be induced by LPS-activated platelets [14] and by antineutrophil autoantibodies isolated from patients with SVV [16], whereas impaired degradation of NETs has been associated with systemic lupus erythematosus as well [20].

In spite of the accumulating evidence for the physiological relevance of NETs, the interrelations of the different subcellular events in NETosis remain elusive. Therefore, a comparative study of neutrophils using high-resolution live-cell imaging was set up to analyze the potential interplay between reactive oxygen species (ROS) generation, mitochondrial membrane potential, intracellular chromatin decondensation and several morphological features, such as massive vacuolization. Moreover, the functional contribution of these subcellular events to NETosis was studied by using pharmacological inhibitors and cells from CGD patients.

\section{Results}

PMA induces typical features of NETosis, which differs both biochemically and morphologically from apoptosis and necrosis

Phorbol myristate acetate stimulation of human neutrophils resulted in the formation of NETs (Supplementary information, Figure $\mathrm{S} 1$ ), as reported previously $[3,6,16$, $18,21]$. In contrast to constitutive or anti-Fas-induced neutrophil apoptosis, PMA-induced NETosis was insensitive to benzyloxycarbonyl-Val-Ala-Asp(Ome)fluoromethylketone (zVAD-fmk) (Supplementary information, Figure S2A). This confirms a previous report that excluded the involvement of caspases in NETosis [6]. This confirmation is further supported by the absence of DEVD-ase activity after PMA stimulation (Supplementary information, Figure S2B). Inhibition of programmed necrosis by pretreatment with $\mathrm{Nec}-1$, an inhibitor of RIP1 kinase activity [22], also did not affect the kinetics of PMA-induced NETosis (Supplementary information, Figure S2C). Live cell imaging of healthy neutrophils stimulated with $100 \mathrm{nM}$ PMA reveals a cell death program characterized by immediate cell flattening and increased adherence, followed by loss of mitochondrial membrane potential and induction of massive vacuolization within approximately $30 \mathrm{~min}$ (Figure 1A; Supplementary information, Video S1). Vacuolization is observed for up to 90-130 min after PMA stimulation, until the nuclear envelope disintegrates and nuclear chromatin decondenses, which allows it to mix with the cytoplasmic content. During all that time, plasma membrane integrity is preserved. Within $40 \mathrm{~min}$ later, the plasma membrane permeabilizes and decondensed chromatin is released. Figure 1B shows the mean percentage of cells undergoing these different subcellular events from a kinetic analysis of 150 cells from four independent experiments. To examine whether loss of mitochondrial membrane potential is sufficient to induce NETosis or possibly affects PMA-induced NETosis, we treated neutrophils with the protonophore carbonyl cyanide 3-chlorophenylhydrazone (CCCP) and the complex III inhibitor antimycin A. Both $\mathrm{CCCP}$ and antimycin A are known to dissipate the mitochondrial membrane potential in neutrophils [23]. Incubation with these agents alone did not induce NET formation and did not affect PMA-induced cell death kinetics (Supplementary information, Figure S3), which suggests that mitochondrial depolarization is not a crucial mediator of DNA decondensation. In this regard, it is noteworthy that neutrophils depend on glycolysis instead of oxidative phosphorylation to meet their energy demands $[23,24]$. This is in agreement with the profound decrease in the number of mitochondria during 

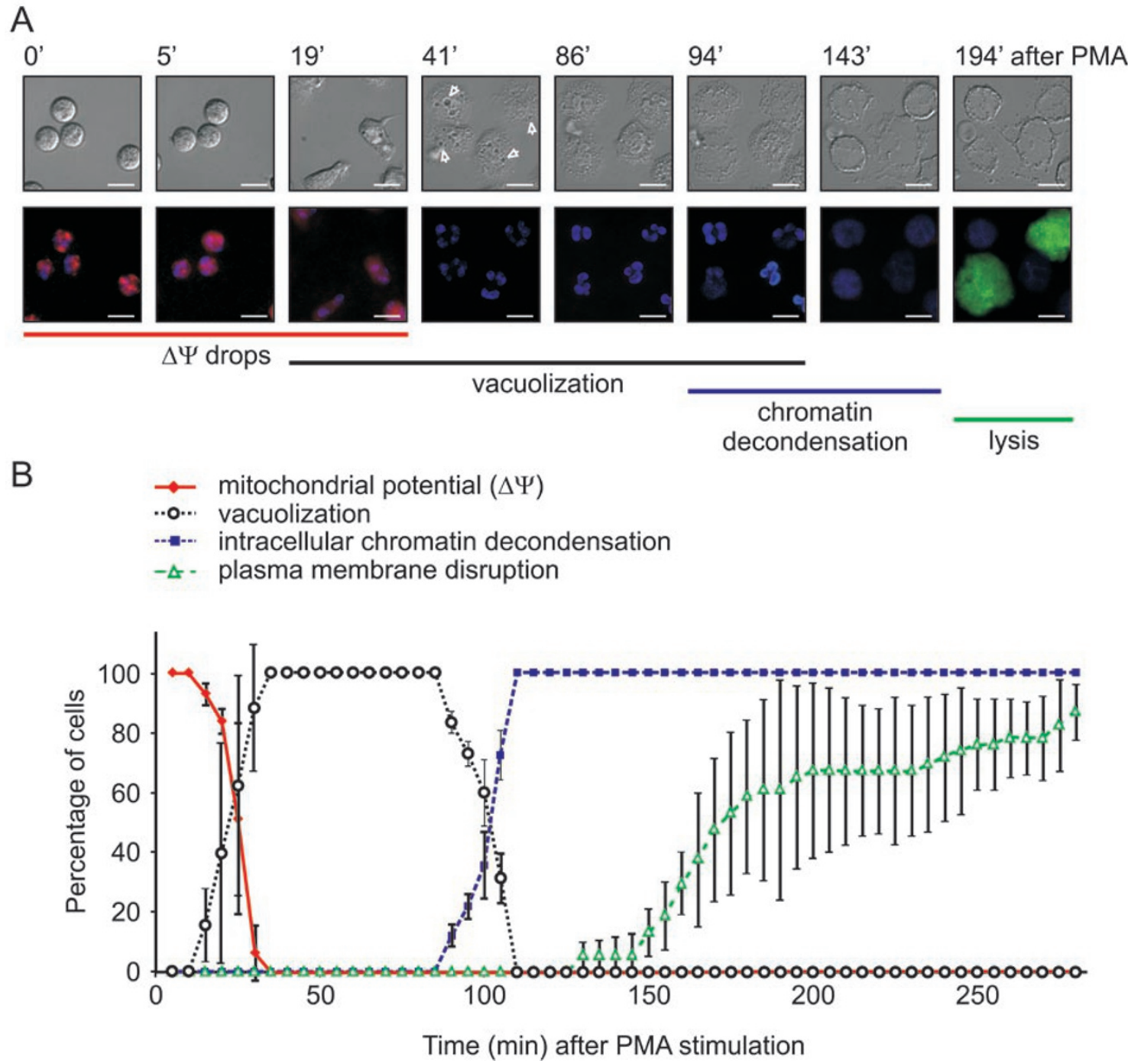

Figure 1 PMA-induced NETosis is characterized, chronologically, by cell flattening and adherence, a drop in mitochondrial membrane potential, vacuolization and intracellular chromatin decondensation. $(A)$ Isolated neutrophils $\left(15 \times 10^{4} \mathrm{PMN}\right.$ per $\mathrm{ml}$ ) were monitored by live cell imaging for four parameters: morphology using differential interface contrast (DIC), mitochondrial potential using TMRM (red), cell death using the cell-impermeable DNA dye Sytox Green (green) and chromatin decondensation using the cell-permeable DNA marker Hoechst 33342 (blue). These were performed in a humidified atmosphere containing $5 \% \mathrm{CO}_{2}$ at $37{ }^{\circ} \mathrm{C}$. Cells were stimulated with $100 \mathrm{nM} \mathrm{PMA}$ and monitored every min for up to 300 min. Important time points are shown in min. Vacuoles are indicated by arrows, and scale bars represent $10 \mu \mathrm{m}$. Results are representative of at least four independent experiments. (B) Kinetic analysis of 150 cells from four independent experiments. Shown is the mean \pm SD of the percentage of cells undergoing subcellular events associated with cell death (loss of mitochondrial potential, vacuolization, intracellular chromatin decondensation and plasma membrane permeabilization).

differentiation of precursor cells into neutrophils [25].

\section{PMA-induced chromatin decondensation requires Nox2 activity}

Stimulation with PMA is known to activate Nox2, which generates superoxide (Supplementary information, Figure S4A). This is of relevance because ROS generation has been reported to be an absolute requisite for the induction of NETosis [6]. However, when NADPH oxidase activity is inhibited by DPI (Supplementary information, Figure S4B) or is absent due to a genetic defect in Nox2 subunits (Supplementary information, Figure S4C and S4D), PMA still induces cell death, albeit with delayed kinetics as compared to normal neutrophils (Figure 2A; Supplementary information, Figure S5). Unstimulated neutrophils from CGD patients or healthy 
volunteers, whether or not pretreated with DPI, die spontaneously from apoptosis [26], but more slowly than in PMA-induced NETosis (Figure 2A). Live cell imaging of the subcellular events described above revealed that fol- lowing PMA stimulation, CGD neutrophils, like normal neutrophils, undergo massive vacuolization and lose their mitochondrial potential quickly (Figures $2 \mathrm{~B}$ and $1 \mathrm{~A}$ ). However, no intracellular chromatin decondensation was

A

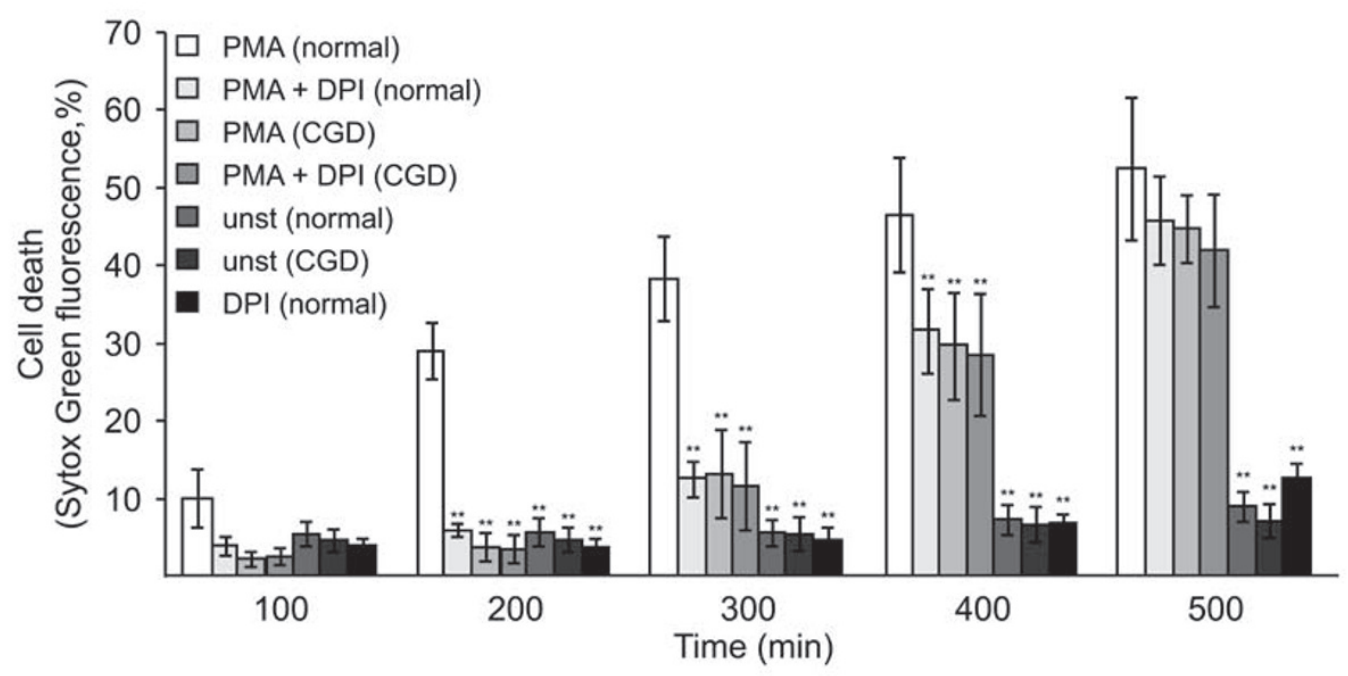

B

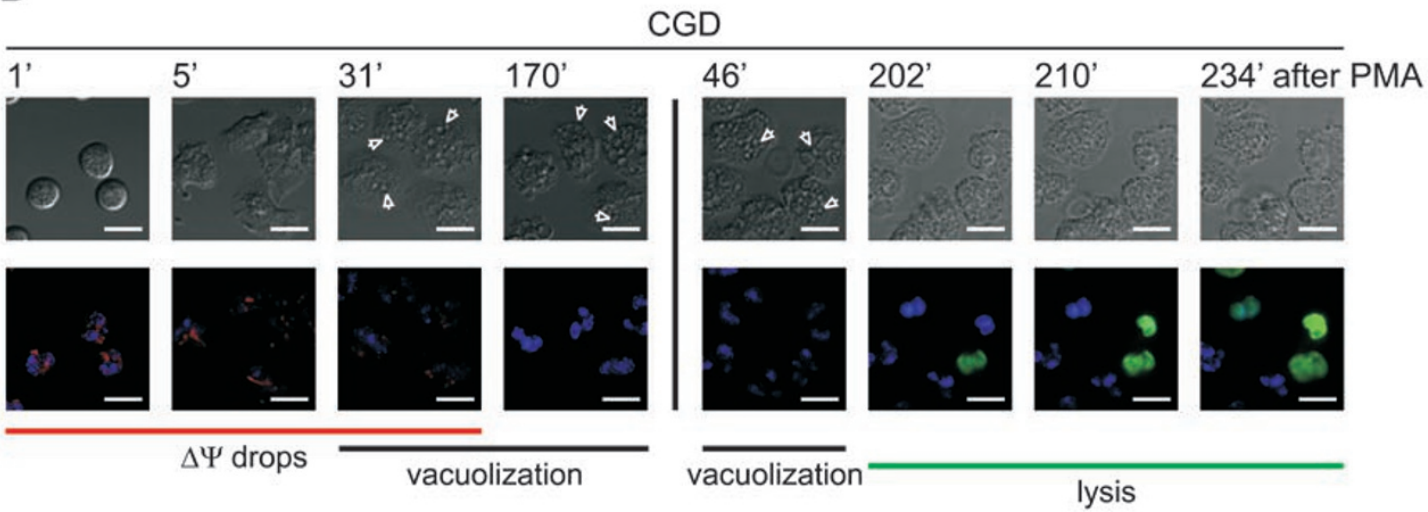

C

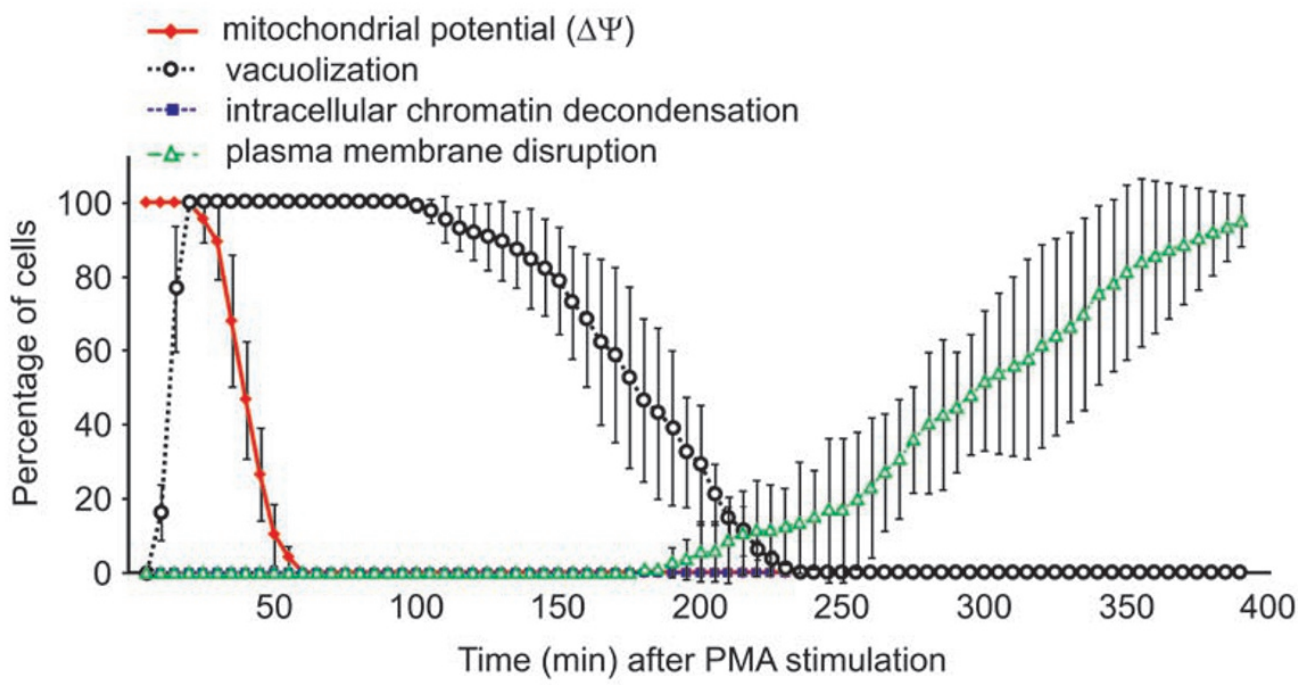


observed in CGD neutrophils at any time between PMA stimulation and plasma membrane disintegration (Figure $2 \mathrm{~B}$ and $2 \mathrm{C}$ ). The massive vacuolization without disintegration of the nuclear envelope seen in CGD neutrophils after PMA stimulation (Figure 2B and 2C) disfavors the proposed causative link between the disintegration of the nuclear envelope and consequent chromatin decondensation on the one hand, and the process of vacuolization on the other hand $[6,9]$. Our observations, therefore, indicate that mechanisms other than nuclear envelope disintegration might contribute to the formation of vesicles and vacuoles with double membranes.

PMA-induced vacuolization involves autophagy and occurs independently of Nox 2 activity

We hypothesized that the numerous vesicles might originate from induction of autophagy because many cell types exhibit massive vacuolization during autophagy-associated cell death $[27,28]$. In agreement with the massive vacuolization on PMA stimulation, which was observed by live cell imaging (Figure 1; Supplementary information, Video S1), transmission electron microscopic analysis of neutrophils revealed the presence of vesicles with double membranes (Figure 3A). These vesicles are not observed in unstimulated neutrophils, which are characterized by multilobulated nuclei and different types of granules, all of which are surrounded by a single phospholipid bilayer. Within 15 min after PMA stimulation, premature autophagosome structures are observed engulfing abundant granules and ribosomes (Figure 3A). After fusion of autophagosomes with endosomes/lysosomes, the autophagic cargo is degraded in the autophagolysosome, which consequently becomes less electron dense (Figure 3B). About 80 min after PMA stimulation, a different type of vesicle with a double phospholipid bilayer appears. The high electron density of these vesicles suggests that they are not autophagosomes (Figure 3A) but might be remnants of the nuclear envelope, as proposed by Fuchs et al. [6]. At this stage, the number of classic granules decrease. Finally, 120 min after stimulation, the nuclear chromatin and granule contents are mixed, and classic granules are no longer observed (Figure 3A). To further characterize these double-membrane vesicles as autophagic vesicles, we monitored the localization of LC3, a marker of autophagy [29]. During the early stages of autophagy induction, LC3 translocates from the cytosol to newly formed autophagosomes. Indeed, within 15 min of PMA stimulation, homogeneously dispersed LC3 shows a punctate distribution pattern that is typical of autophagy. Interestingly, translocation of endogenous LC3 is observed in both normal and CGD neutrophils (Figure 3C; Supplementary information, Videos S2-S5). These results fit with the PMA-induced vacuolization of CGD neutrophils observed by live cell imaging (Figure $2 \mathrm{~B}$ and $2 \mathrm{C}$ ) and indicate that PMA induces autophagy in a superoxide-independent manner. We also measured the autophagic flux, which is important for distinguishing increased autophagosome formation from impaired degradation/maturation. Pretreatment with the vacuolar $\mathrm{H}+$-ATPase inhibitor bafilomycin A1 prevents lysosomal acidification [30] and results in the accumulation of autophagosomes and consequent protection of LC3 from degradation. Stimulation of CGD or normal neutrophils with PMA for 30 min resulted in a decrease in LC3 levels, and bafilomycin A1 pretreatment significantly prevented PMA-induced LC3 degradation (Supplementary information, Figure S6). These results, together with the observation of mature autophagolysosomes by EM (Figure $3 \mathrm{~A}$ ), indicate that PMA induces the formation and maturation of autophagosomes.

\section{Inhibition of either Nox2 activity or autophagy prevents PMA-induced NETosis and stimulates apoptosis}

To further examine the role of PMA-induced autophagy

Figure 2 Absence of Nox2 activity blocks PMA-induced intracellular chromatin decondensation and delays cell death, whereas vacuolization and the drop in mitochondrial membrane potential are unaffected. (A) Normal and CGD neutrophils $\left(2 \times 10^{5}\right)$ were incubated with or without $10 \mu \mathrm{M} \mathrm{DPI}$ for $30 \mathrm{~min}$, and either left unstimulated or were stimulated with $100 \mathrm{nM}$ PMA for the indicated periods in the presence of the cell-impermeable DNA dye Sytox Green (50 nM). Data are expressed as percentage of maximal Sytox Green fluorescence \pm SD $(n=3)$ as a function of time. ${ }^{*} P<0.01$ and ${ }^{* *} P<0.001$, as compared to PMAstimulated normal neutrophils. (B) Isolated neutrophils obtained from a CGD patient were examined by live cell imaging for different parameters in a humidified atmosphere containing $5 \% \mathrm{CO}_{2}$ at $37{ }^{\circ} \mathrm{C}$. Left panel: morphology using DIC, mitochondrial potential using TMRM (red), and chromatin decondensation using the cell-permeable DNA marker Hoechst 33342 (blue). Right panel: morphology using DIC, chromatin decondensation using the cell-permeable DNA marker Hoechst 33342 (blue) and cell death using the cell-impermeable DNA dye PI (green). Cells were stimulated with 100 nM PMA and monitored every min for up to 400 min. Important time points are shown in min. Vacuoles are indicated by arrows, and scale bars represent 10 $\mu \mathrm{m}$. (C) Kinetic analysis of 85 cells from three independent experiments. Shown is the mean \pm SD of the percentage of cells undergoing subcellular events associated with cell death (loss of mitochondrial potential, vacuolization, intracellular chromatin decondensation and plasma membrane permeabilization). 
A

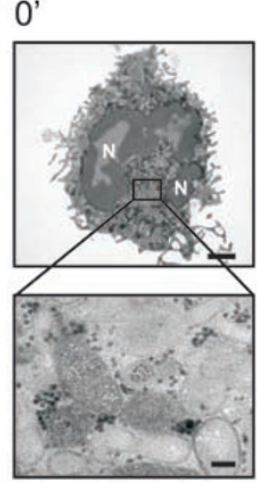

$15^{\prime}$

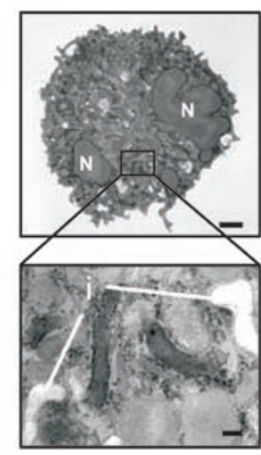

$30^{\prime}$

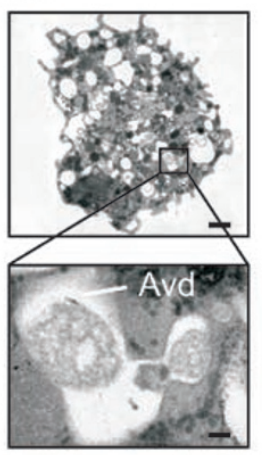

$80^{\prime}$

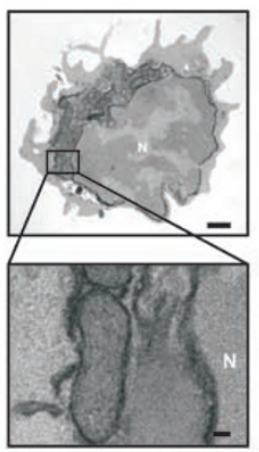

120' after PMA

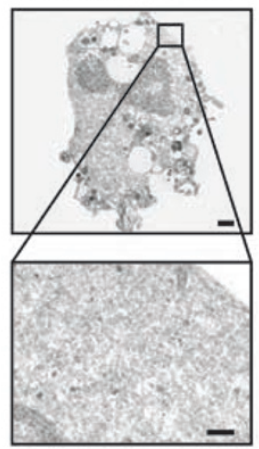

B

\section{0 ' after PMA}

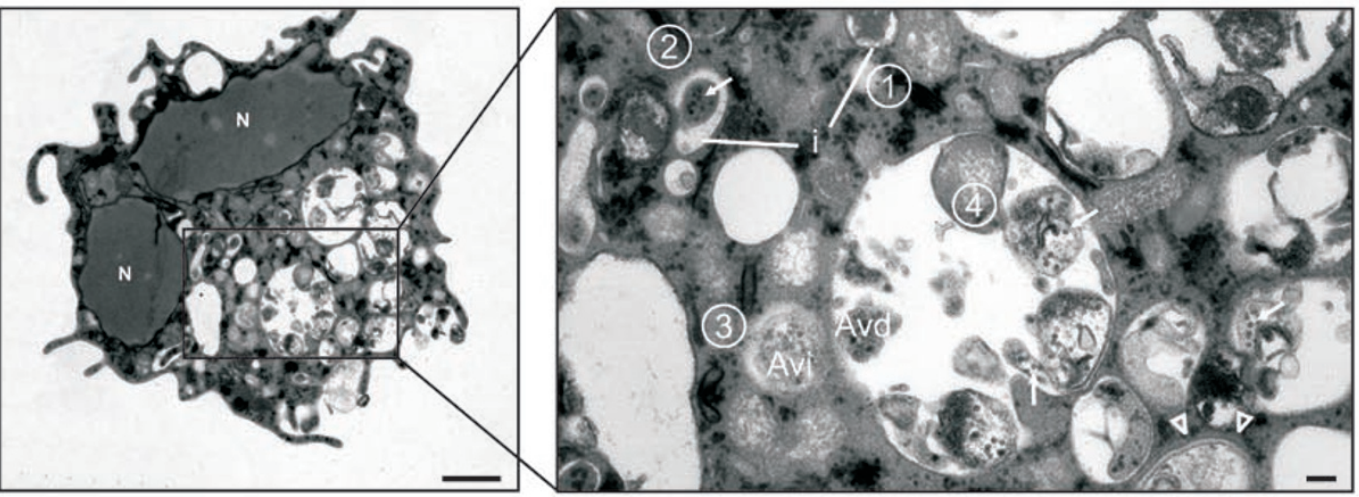

C

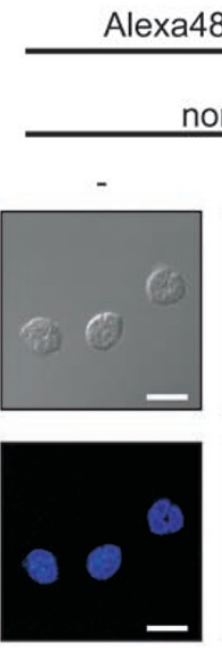

PMA
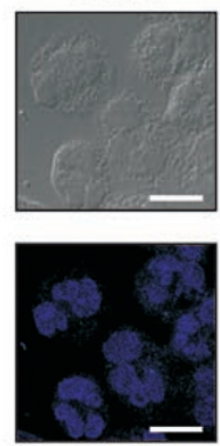

Anti-LC3 + Alexa488-sec.Ab
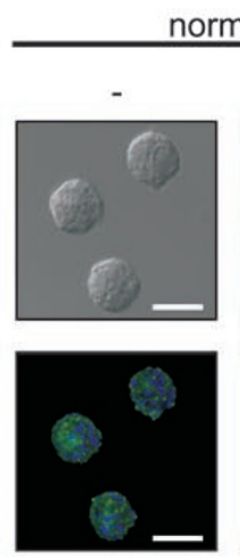

ormal

PMA
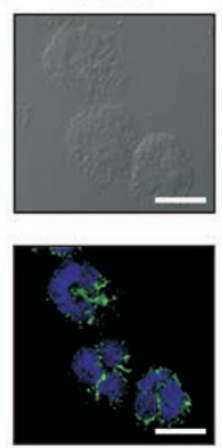

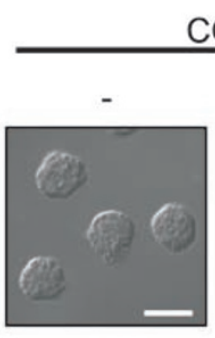

CGD

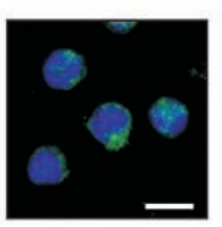

PMA
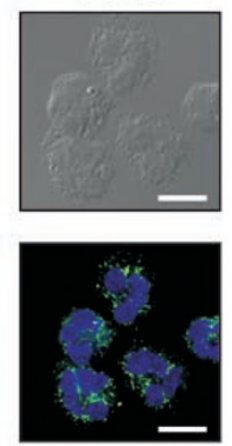

Figure 3 PMA-induced vacuolization is preceded by typical features of autophagy. (A) Human neutrophils $\left(3 \times 10^{5} \mathrm{PMN}\right.$ per $\mathrm{ml}$ ) were left unstimulated or were stimulated with $100 \mathrm{nM}$ PMA for 15, 30, 80 or 120 min. Cells were then fixed and examined by transmission electron microscopy (TEM). (B) Zoom in on TEM picture of human neutrophils stimulated with $100 \mathrm{nM}$ PMA for $30 \mathrm{~min}$. De novo formation of isolation membranes (i) (1). Engulfment of cytoplasmic content (2), such as granules or ribosomes (arrows). Generation of early immature autophagic vacuoles (Avi) (3) characterized by a double phospholipid bilayer (arrowheads). Degradation of vacuolar content on fusion with endosomes/lysosomes in late degradative autophagic vacuole (Avd) (4). (C) PMA stimulation induces recruitment of LC3 to autophagosomes. Normal neutrophils and neutrophils from CGD patients were left unstimulated or were stimulated for 15 min with $100 \mathrm{nM}$ PMA and then fixed, permeabilized and stained for LC3 and for nuclear chromatin, and analyzed by confocal microscopy. DIC and fluorescent images are shown. Scale bars represent $10 \mu \mathrm{m}$. Representative results of three independent experiments are shown. In (A and $\mathbf{B})$ the scale bars for full cell images indicate $1 \mu \mathrm{m}$ and for magnified cell areas $100 \mathrm{~nm}$. Nuclear lobi are indicated $(\mathrm{N})$. 
in NETosis, we used the PI3K inhibitor wortmannin, which inhibits autophagy [31,32]. Wortmannin treatment alone did not affect the kinetics (Figure 4C) or morphological changes (Supplementary information, Video S6) of spontaneous neutrophil apoptosis at early time points, as reported previously [26]. As confirmed by live cell imaging, freshly isolated neutrophils pretreated with 100 nM wortmannin no longer show massive vacuolization on PMA stimulation, which further underscores the idea that the massive vacuolization is linked to autophagy induction (Figure 4; Supplementary information, Video S7). Pretreatment with $100 \mathrm{nM}$ wortmannin did not affect PMA-induced superoxide production, as shown by live cell imaging using 5-(and-6)-carboxy-2',7'dichlorofluorescein diacetate (DCFDA), an ROS-sensitive probe (Figure 4A), and as measured by lucigenin chemiluminescence (Figure 4B). Importantly, although Nox2 activity remained intact when autophagy was pharmacologically blocked (Figure 4A), PMA did not induce chromatin decondensation (Figure 4A; Supplementary information, Video S7), but the cell death rate was not affected (Figure 4C). In contrast, Nox2 inhibition led to retardation of the cell death process, as previously mentioned (Figure 2A). However, when we analyzed the cell death process in more detail, we observed that inhibition of either Nox 2 or autophagy led to activation of caspases (as measured by DEVDase activity) and to the appearance of morphological features of apoptosis, such as membrane blebbing (Figure 5; Supplementary information, Videos S8 and S9). These events did not occur at any time in normal neutrophils stimulated with PMA (Figure 1; Supplementary information, Figure S1 and Video S1). In conclusion, these results show that inhibition of either autophagy or Nox 2 prevents chromatin decondensation, which is essential for NETosis and NET formation [7], and results in a type of cell death characterized by hallmarks of apoptosis. These results indicate that apoptosis might function as a backup program for NETosis when autophagy or Nox2 activity is prevented.

\section{Discussion}

NETosis is characterized by decondensation of intracellular chromatin and disintegration of the nuclear envelope, which allows mixing of the chromatin with cationic antimicrobial molecules originating from the granules. When the plasma membrane finally permeabilizes, the microbicidal extracellular traps are released. In this article we demonstrate that neither inhibition of caspases by zVAD-fmk [6] nor inhibition of RIP1 kinases by necrostatin-1 affects NETosis. Thus, NETosis should be considered a distinct cell death program. It was recently shown that decondensation of nuclear chromatin preceding NET formation is mediated by histone citrullination $[7,10]$. The inability of CGD neutrophils to generate NETs in response to PMA indicates that Nox2 activity is essential for the intracellular chromatin decondensation that precedes NET formation [6], but this was not demonstrated directly. We demonstrate that Nox 2 activity is indeed required for chromatin decondensation in intact neutrophils. We also observed that PMA-induced NETosis is associated with induction of autophagy, despite the view that autophagy might not occur in neutrophils [33]. Autophagy is a well-conserved, essential, intracellular degradation process known to regulate protein and organelle turnover in many cells [32]. In addition, autophagy has been implicated in cell death as well [28]. As for neutrophil cell death, von Gunten et al. [34] originally observed autophagosome-like structures during nonapoptotic cell death induced by anti-Siglec 9 antibodies after priming of neutrophils with proinflammatory cytokines such as GM-CSF, IFN- $\alpha$ and IFN- $\gamma$. Autophagy was recently confirmed in murine neutrophils by two independent groups as well as in human neutrophils $[35,36]$. Of interest, during the preparation of this article, Mitroulis et al. [37] also reported that autophagy can be observed in human neutrophils in response to PMA stimulation. However, these reports did not investigate autophagy in the context of neutrophil cell death [35-37]. We investigated the extent to which autophagy depends on superoxide production, and whether autophagy induction is implicated in NETosis. Superoxide is the major type of ROS known to stimulate autophagy [38]. Surprisingly, we observed that PMA induces autophagy in CGD neutrophils, which are devoid of Nox2 activity. These results demonstrate that PMA-induced autophagy does not require Nox2 activity per se.

We next examined the extent to which induced autophagy affects events essential for NETosis, such as Nox2 activity and intracellular chromatin decondensation. Pharmacological inhibition of autophagy by wortmannin prevented the induction of vacuolization but did not affect superoxide production. This inhibition of autophagy markedly prevented intracellular chromatin decondensation without affecting Nox 2 activity. This demonstrates that Nox 2 activity is necessary but not sufficient for the intracellular chromatin decondensation implicated in NET formation. Moreover, induced autophagy alone, which is observed in CGD neutrophils lacking Nox2 activity, is also not sufficient to induce intracellular chromatin decondensation. These results demonstrate that a combination of autophagy and superoxide production is necessary for the induction of intracellular chromatin decondensation during PMA-induced NETosis. 
$A_{1}$,
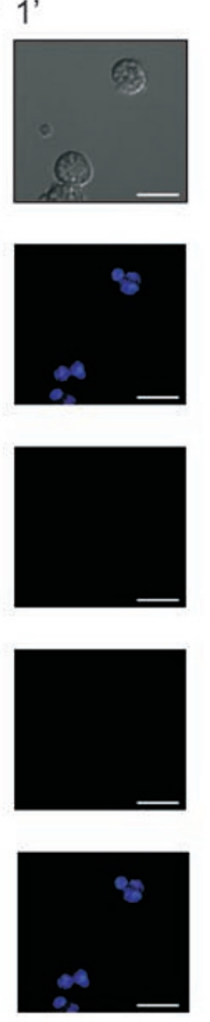

$20^{\prime}$
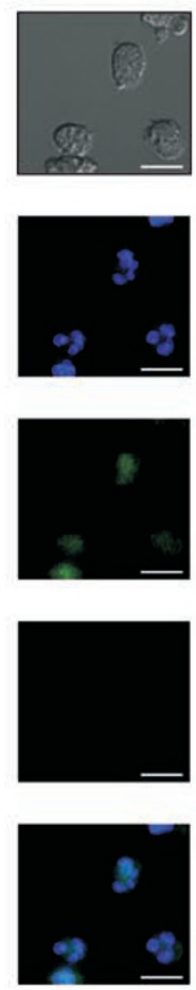

$63^{\prime}$
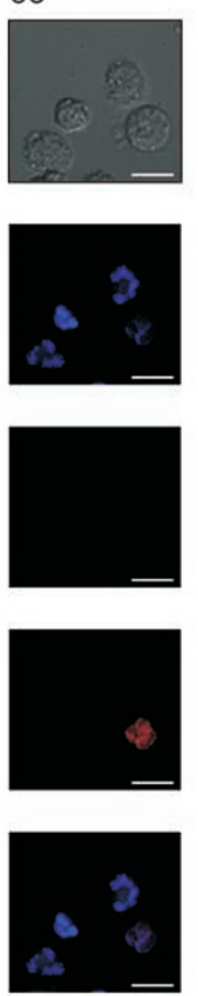

$87^{\prime}$
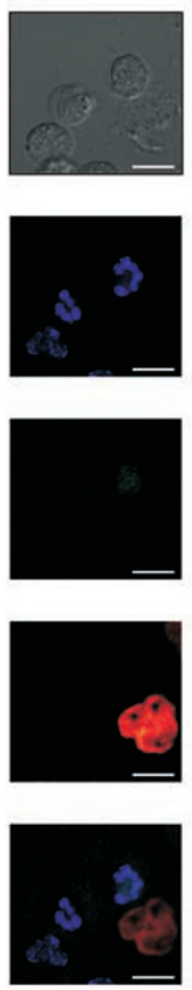

99' after PMA

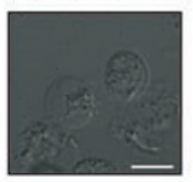

DIC

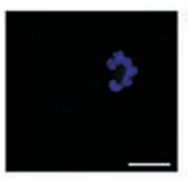

Hoechst 33342

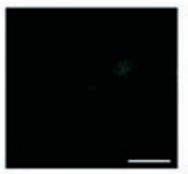

DCFDA

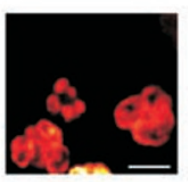

PI

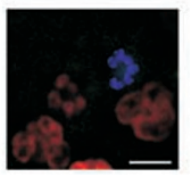

merge
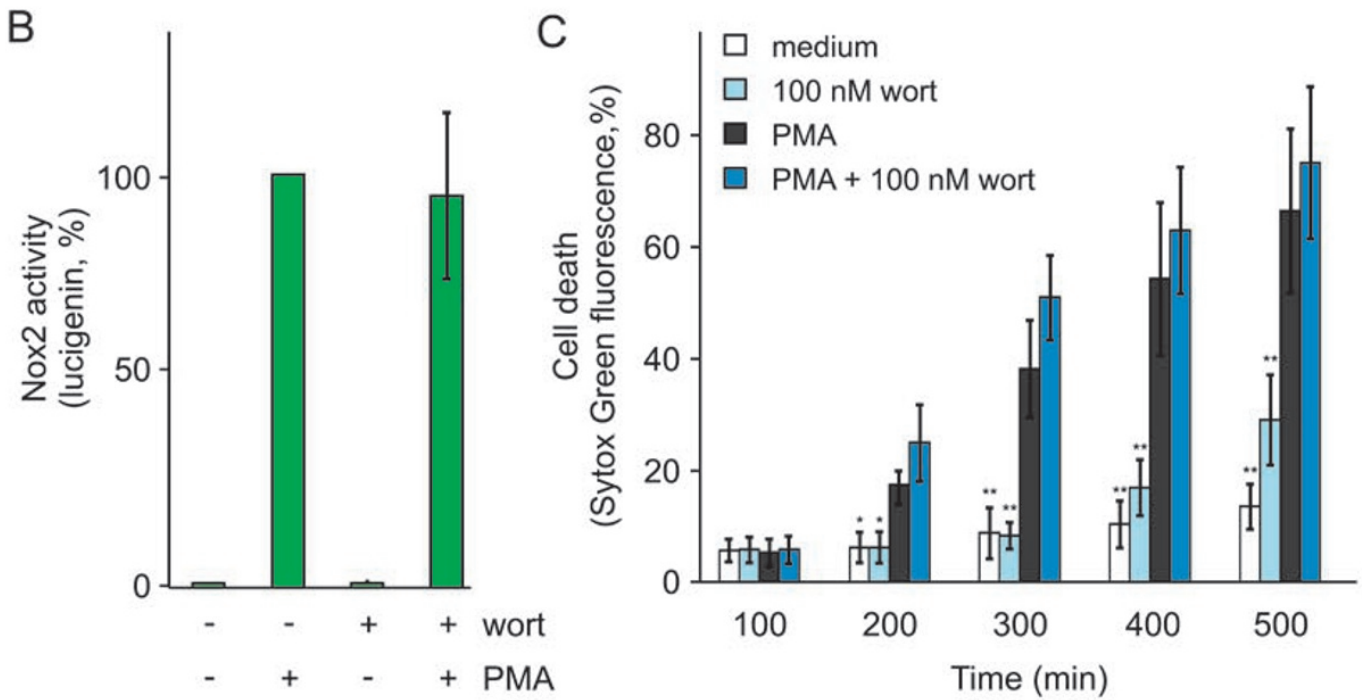

Figure 4 Inhibition of autophagy by wortmannin blocks vacuolization and intracellular chromatin decondensation, but generation of ROS is unaffected. (A) Human neutrophils were incubated with the ROS probe DCFDA (green) and the cell-permeable DNA dye Hoechst 33342 (blue) for $30 \mathrm{~min}$ at $37{ }^{\circ} \mathrm{C}$ in a humidified atmosphere containing $5 \% \mathrm{CO}_{2}$. Subsequently, medium containing the cell-impermeable DNA marker PI (red) and wortmannin (100 nM) was refreshed. Cells were stimulated with $100 \mathrm{nM}$ PMA and monitored every min by live cell imaging. Important time points are shown in min. Representative results of three independent experiments are shown. The scale bars represent $10 \mu \mathrm{m}$. (B) Cells were either untreated or pretreated with wortmannin (100 nM) for $30 \mathrm{~min}$, and then stimulated with $100 \mathrm{nM}$ PMA. Superoxide production was determined by enhanced chemiluminescence. Mean maximal superoxide production is expressed as percentage of control \pm SD $(n=3)$. (C) In the presence of $50 \mathrm{nM}$ Sytox Green, $2 \times 10^{5}$ neutrophils were incubated with or without wortmannin (100 nM) for $30 \mathrm{~min}$ and were either left unstimulated or stimulated with $100 \mathrm{nM}$ PMA for the indicated periods. Cell death was detected by measuring the fluorescence of the cell-impermeable DNA dye Sytox Green. Data are expressed as percentage of maximal Sytox Green fluorescence $\pm \operatorname{SD}(n=3)$ as a function of time. ${ }^{*} P<0.05$ and ${ }^{* *} P<0.01$, as compared to PMA-stimulated normal neutrophils. 
A
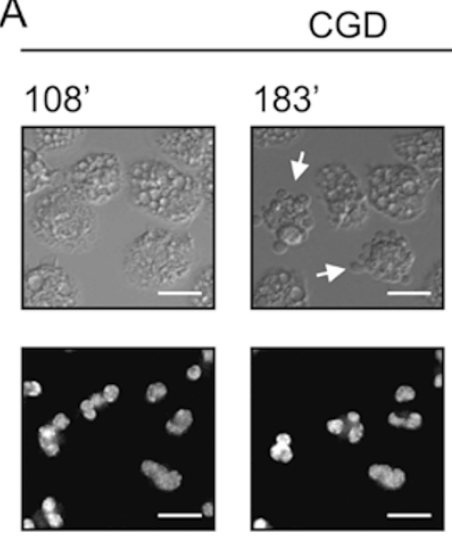

C

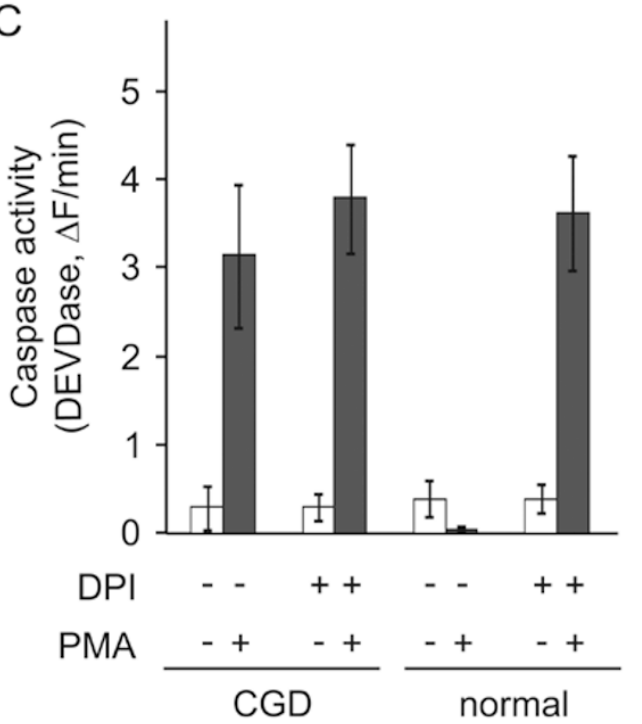

B
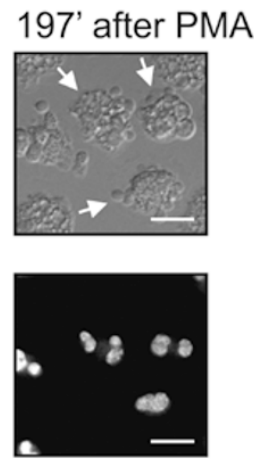

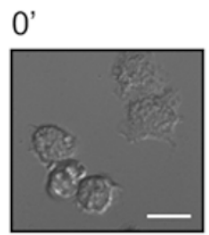

wortmannin

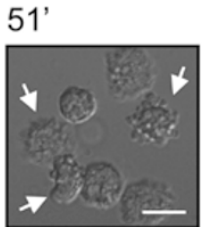

83' after PMA
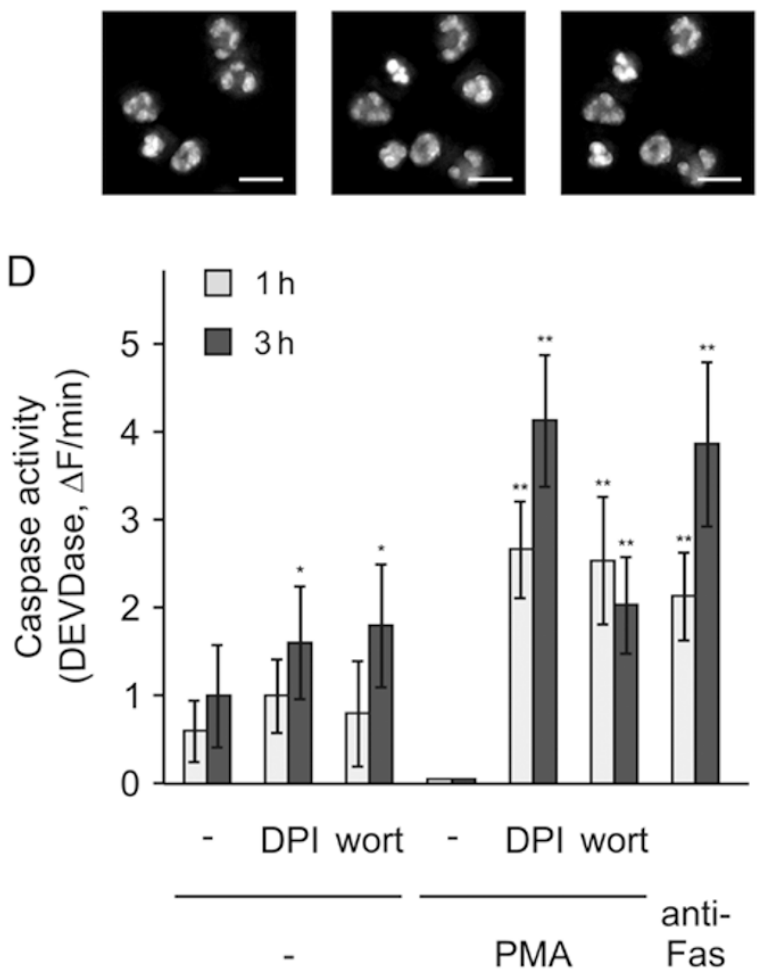

Figure 5 PMA induces features of apoptosis when Nox2 or autophagy is inhibited. (A) Live cell images of neutrophils isolated from a CGD patient and stimulated with 100 nM PMA. Morphology was examined using DIC and chromatin decondensation using the cell-permeable DNA marker Hoechst 33342 (blue). Cells were monitored every min for up to 300 min, and important time points are shown in min. PMA stimulation of CGD neutrophils induced membrane blebbing (arrows). (B) Live cell images of isolated neutrophils pretreated with wortmannin (100 nM) for $30 \mathrm{~min}$. Morphology was examined using DIC and chromatin decondensation using the cell-permeable DNA marker Hoechst 33342 (blue). PMA-induced membrane blebbing (arrows) in normal neutrophils pretreated with wortmannin. (C) Normal and CGD neutrophils were incubated with or without DPI $(10 \mu \mathrm{M})$ for $30 \mathrm{~min}$. Samples were collected (white bars) and the remainder was further stimulated with $100 \mathrm{nM}$ PMA for $3 \mathrm{~h}$ (gray bars). Protease activity against the caspase substrate DEVD-amc was determined. Data are expressed as the mean $\Delta \mathrm{F} / \mathrm{min} \pm \mathrm{SD}(n=3)$. (D) Normal neutrophils were incubated for 30 min with $10 \mu \mathrm{M}$ DPI or $100 \mathrm{nM}$ wortmannin (wort) or left untreated. Subsequently, cells were further stimulated for $1 \mathrm{~h}$ (light gray bars) or $3 \mathrm{~h}$ (dark gray bars) with PMA (100 nM) or with agonistic anti-Fas antibody $(250 \mathrm{ng} / \mathrm{ml})$ and analyzed for DEVDase activity. Data are expressed as the $\mathrm{mean} \Delta \mathrm{F} / \mathrm{min} \pm$ SD $(n=3)$. ${ }^{*} P<0.01$ and ${ }^{* *} P<0.001$, as compared to PMA-stimulated neutrophils.

Because intracellular chromatin decondensation is crucial for NETosis, we wondered whether its absence affects the cell death program itself. Interestingly, when either autophagy or Nox2 activity was blocked, PMA induced a type of cell death characterized by membrane blebbing and caspase activity, which are features of apoptosis. Consequently, when intracellular chromatin decondensation during NETosis was prevented, an alternative apoptotic cell death pathway came into play. This pathway also eventually resulted in chromatin decondensation, but only after plasma membrane permeabilization during post-apoptotic secondary necrosis. Therefore, the chromatin decondensation that follows spontaneous apoptosis of neutrophils ex vivo [26] should not be con- 
fused with proper NET formation during NETosis.

Our results demonstrate that both autophagy and Nox2 activity are needed to activate NETosis fully but they might also prevent apoptosis. Indeed, Hampton and coworkers [39-41] have repeatedly shown the ability of activated Nox 2 to inhibit caspases. So, we were surprised to observe membrane blebbing and caspase activity in wortmannin-treated neutrophils stimulated with PMA, because NADPH oxidase activity remained unaffected. We propose that both Nox 2 activity and autophagy are required to inactivate caspases. Indeed, stimulated CGD neutrophils lacking NADPH oxidase activity still undergo autophagy, show caspase activity and die by apoptosis. In addition, when autophagy is blocked in stimulated normal neutrophils, caspase activity and apoptosis become apparent. These results are in line with the induction of apoptosis observed in mammalian cell lines when starvation-induced autophagy was inhibited [42]. Both prosurvival and prodeath functions have been ascribed to autophagy [43]. A criterion to claim autophagic cell death is to observe decreased cell death when autophagy is inhibited [43]. When neutrophil autophagy was pharmacologically inhibited, we observed impaired intracellular chromatin decondensation, but the neutrophils continued to die though with features of apoptosis. This suggests that autophagy is required for the initial phase of NETosis (DNA decondensation) rather than for the cell death process itself. Our results using necrostatin-1 [22] also demonstrate that caspase-independent cell death mediated by RIP1 kinase activity is not implicated in PMAinduced NETosis, which excludes its role in this type of neutrophil cell death.

Bacterial challenge is known to induce Nox2 activation [27], but it can also induce autophagy [35, 36]. It was recently shown that engagement of Toll-like receptor and $\mathrm{Fc}$ receptor signaling during phagocytosis induces autophagy in primary neutrophils [36]. Moreover, in line with our results, when primary neutrophils are challenged with Staphylococcus, caspases are inactivated [41] and NETs are formed [6].

A previous observation of CGD neutrophils generating NETs in response to glucose oxidase (GO)-derived ROS, but not to PMA, suggested that ROS alone might be sufficient to induce NETosis [7]. We therefore examined $\mathrm{H}_{2} \mathrm{O}_{2}$-induced death of human neutrophils. In this regard, it should be noted that direct stimulation with $\mathrm{H}_{2} \mathrm{O}_{2}$ is more transient than the continuous production of ROS by GO. Consequently, it is conceivable that direct stimulation with $\mathrm{H}_{2} \mathrm{O}_{2}$ mimics the Nox2-dependent oxidative burst more closely than GO-derived ROS production. In addition, the GO used to induce NETosis was produced in Aspergillus niger [6]. Because Aspergillus species have been shown to induce NETosis [18], we cannot rule out the possibility that nonmammalian compounds in GO solutions provided the additional triggers necessary to induce NETosis during GO stimulation. Noteworthy, it has been shown that $\mathrm{H}_{2} \mathrm{O}_{2}$ is not the major ROS regulating autophagy [38], and that it might not even induce autophagy [44]. In agreement with these results, we could not detect any noteworthy autophagy in $\mathrm{H}_{2} \mathrm{O}_{2}$ stimulated neutrophils (Supplementary information, Figure S7). Live cell imaging revealed that $\mathrm{H}_{2} \mathrm{O}_{2}$ did not induce NETosis, as shown by the absence of cell flattening, adherence, massive vacuolization and intracellular chromatin decondensation in intact cells. At lower millimolar concentrations well below $8 \mathrm{mM}, \mathrm{H}_{2} \mathrm{O}_{2}$ induced an apoptotic cell death morphology (Supplementary information, Figure S7A and Video S10), whereas at concentrations above $8 \mathrm{mM}$, which are not physiological, it induced a necrotic cell death morphology (Supplementary information, Figure S7B and Video S11). These results further support our previous conclusion that ROS generation is insufficient to induce intracellular chromatin decondensation and subsequent NET formation. A nonsufficient role of ROS in NETosis is further supported by the inability of formyl-methionyl-leucyl-phenylalanine (fMLP, a potent inducer of Nox2 activity [26]) to induce NETosis (unpublished observations), presumably due to activation of signaling pathways known to inhibit apoptosis and autophagy $[1,31,45]$. In addition, stimulation of neonate neutrophils with PMA results in ROS production but not in NET formation, in contrast to PMA stimulation of adult neutrophils [21].

Of interest, LPS and IL-8 have been reported to induce NET formation [3]. In contrast, we only observed a delay of apoptosis by stimulation with either LPS [26] or IL-8 (Supplementary information, Video S12). These results are in line with those of others who also did not observe NET formation in response to LPS [14]. Instead, our observations and those of others suggest that LPS and IL-8 protect neutrophils against apoptosis $[1,26]$. Of interest, both LPS and IL- 8 sensitize Nox 2 activity by a process called priming, but they cannot induce Nox2 activity directly [46]. This is relevant, as Nox2 activity is essential for NETosis, as evidenced by the inability of CGD neutrophils to undergo NETosis [6]. The inability of neutrophils from CGD patients to undergo NETosis was recently correlated with their sensitivity to infection [18]. In this regard, it is noteworthy that many other antimicrobial ROS-dependent processes are also prevented in CGD [47]. Recently, stimulation of neutrophils with compounds that sensitize/prime Nox 2 without activating it was also shown to result in the extracellular release of chromatin from viable neutrophils [48]. This chromatin 
was shown to originate exclusively from mitochondria, which is unusual given the low numbers of mitochondria in neutrophils. These results suggest that functional extracellular traps might originate from the activation of a wide range of different signaling pathways and cellular processes.

In conclusion, we demonstrate that intracellular chromatin decondensation and NET formation in PMAstimulated neutrophils are secondary to autophagy and superoxide production, both of which are required but insufficient on their own to mediate NETosis (Figure 6). Moreover, both processes are required to inhibit the activation of caspases and consequent apoptotic cell death (Figure 6). The combined autophagy- and Nox2dependent chromatin decondensation in intact neutrophils and the inhibition of caspases might contribute to the generation of functional NETs. It is conceivable that as the interval between intracellular chromatin deconden- sation and plasma membrane permeabilization lengthens, more intragranular antimicrobial molecules are absorbed by the accessible and voluminous chromatin. In addition, exposure of nuclear histones to the proteolytic activities outside the nucleus might even generate more potent antimicrobial cleavage products, as has been described in fish and amphibians [11], and perhaps the absence of caspase activity is essential in this process. We plan to functionally and biochemically analyze the antimicrobial properties of chromatin released from primary neutrophils after stimulation of different cell death programs in neutrophils.

\section{Materials and Methods}

\section{Reagents and antibodies}

Antimycin A, bafilomycin A1, CCCP, DPI, $\mathrm{H}_{2} \mathrm{O}_{2}$, LPS, PMA, wortmannin, PMSF, Pefabloc-SC and propidium iodide (PI) were

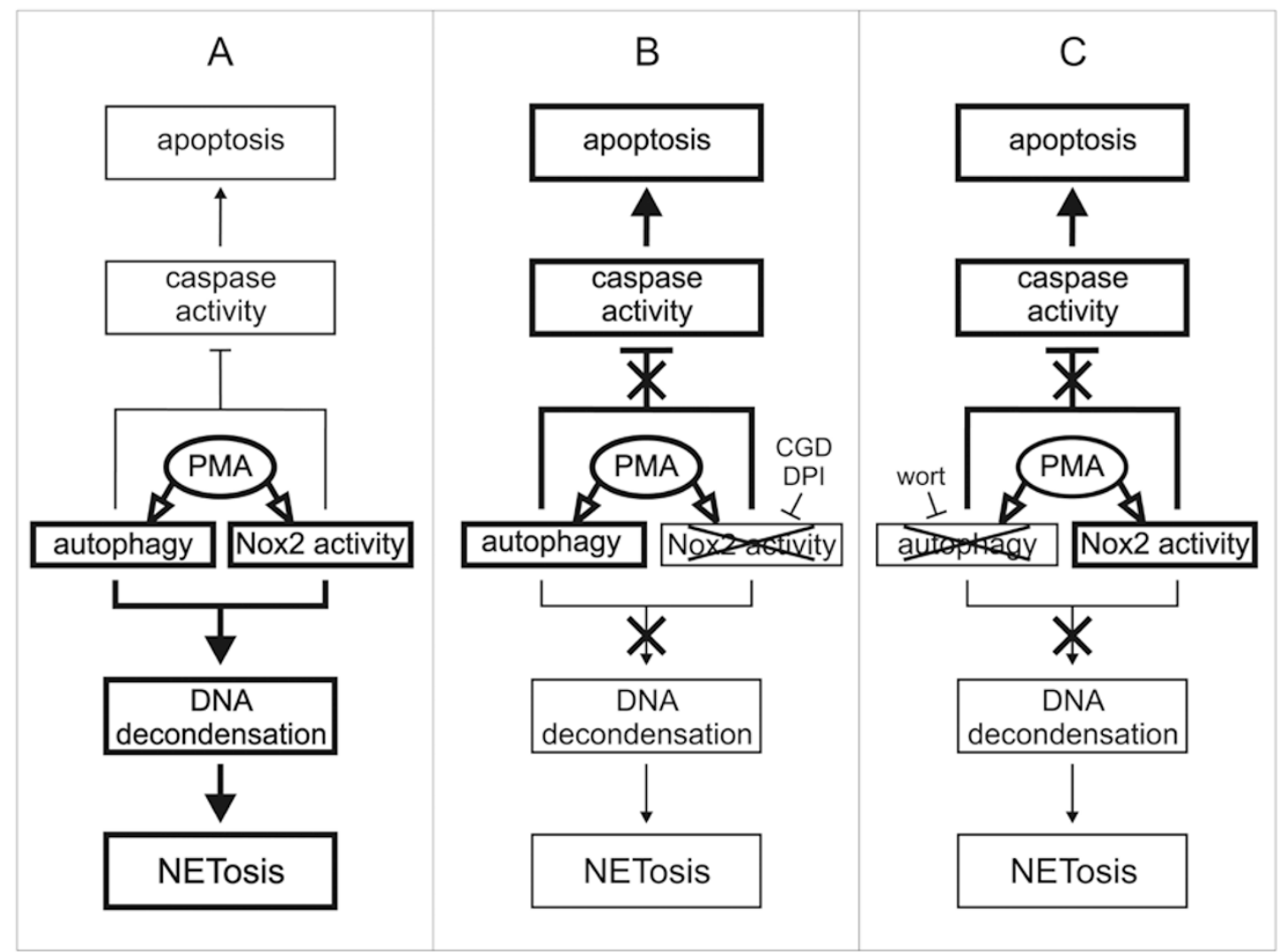

Figure 6 (A) PMA-induced NETosis requires both autophagy and superoxide production, which trigger the intracellular chromatin decondensation preceding NET formation. (B) Pharmacological (DPI) or genetic (CGD) inactivation of Nox2 activity prevented PMA-induced superoxide production, but not PMA-induced autophagy. No intracellular chromatin decondensation occurred, and despite massive vacuolization, cells finally underwent cell death characterized by features of apoptosis. (C) Pharmacological (wortmannin) inhibition of PMA-induced autophagy did not interfere with PMA-induced superoxide production. Nevertheless, intracellular chromatin decondensation did not occur, and cells underwent cell death characterized by features of apoptosis. 
purchased from Sigma-Aldrich (St Louis, MO, USA). Poly-Llysine was purchased from R\&D Systems (Abington, UK). zVADfmk was from Bachem (Bubendorg, Switzerland), and Annexin V-Alexa 488, Sytox Green (SG), Hoechst blue 33342, tetramethylrhodamine (TMRM) and DCFDA were from Molecular Probes (Carlsbad, OR, USA). Anti-human Fas antibody (clone 2R2) was purchased from Cell Diagnostica (Münster, Germany). Monoclonal anti-gp91 ${ }^{\text {phox }}$ antibody (54.1) was purchased from Santa Cruz (Heidelberg, Germany). LC3 antibody with a higher specificity against LC3-II than for LC3-I was purchased from Nanotools (Teningen, Germany). Goat anti-mouse Alexa 488 Ab was purchased from Molecular Probes-Invitrogen. HRP-conjugated antitubulin was purchased from Abcam (Cambridge, UK). IL-8 was a kind gift from Dr Paul Proost (Rega Institute, Leuven, Belgium).

\section{PMN isolation}

Blood samples were collected from healthy volunteers and two CDG patients after obtaining informed consent. Both CDG patients have a defect in gp91 ${ }^{\text {phox }}$. Neither patient suffered from an acute infection at the time blood was drawn. CGD diagnosis was confirmed by measurement of superoxide production after stimulation with $100 \mathrm{nM}$ PMA or $0.5 \mu \mathrm{M}$ fMLP.

Neutrophils were purified by dextran sedimentation, followed by purification on Ficoll-Paque (Pharmacia, Sweden) and hypotonic lysis of contaminating red blood cells [26]. Isolated neutrophils were suspended in RPMI 1640 (Sigma-Aldrich) supplemented with $4 \%$ heat-inactivated fetal calf serum (FCS), $1 \%$ penicillin/ streptomycin and $0.1 \%$ gentamycin, and incubated at $37{ }^{\circ} \mathrm{C}$ in a humidified atmosphere containing $5 \% \mathrm{CO}_{2}$.

\section{Caspase cleavage assay}

Ac-DEVD-amc cleavage was measured by a fluorimetric assay modified as described previously [49]. Neutrophils $\left(5 \times 10^{6}\right.$ PMN per $\mathrm{ml}$ ) were incubated for various durations, after which they were centrifuged at $500 \times \mathrm{g}$ for $5 \mathrm{~min}$. Cell pellets were resuspended in $140 \mu 1$ ice-cold caspase lysis buffer (CLB) (1\% NP40, 10 mM Tris- $\mathrm{HCl}$ (pH 7.4), $200 \mathrm{mM} \mathrm{NaCl}, 5 \mathrm{mM}$ EDTA, 10\% glycerol, $1 \mathrm{mM}$ oxidized glutathione, $1 \mathrm{mM}$ PMSF, $2 \mathrm{mM}$ DFP, $0.3 \mathrm{mM}$ aprotinin, $1 \mathrm{mM}$ leupeptin). Protein concentration was then determined by the BCA method. A sample containing $15 \mu \mathrm{g}$ proteins was suspended in a final volume of $150 \mu \mathrm{l}$ in assay buffer (CLB supplemented with $10 \mathrm{mM}$ DTT and $50 \mu \mathrm{M}$ DEVD-amc). The release of fluorescent 7-amino-4-methylcoumarin was measured every $2 \mathrm{~min}$ for $1 \mathrm{~h}$ in a fluorometer (PerSeptive Biosystems, Cambridge, UK) at an excitation wavelength of $370 \mathrm{~nm}$ and an emission wavelength of $445 \mathrm{~nm}$. The increase in fluorescence was linear. Data are expressed as the increase in fluorescence per minute ( $\Delta$ fluorescence/min).

\section{Cell death assay}

Human neutrophils were seeded at $2 \times 10^{5} \mathrm{PMN}$ per $\mathrm{ml}$ in black flat-bottomed 96-well plates (Greiner, Nurtingen, Germany) in HEPES-buffered RPMI 1640 medium (Sigma-Aldrich) supplemented with $4 \% \mathrm{FCS}, 1 \%$ penicillin/streptomycin, $0.1 \%$ gentamycin and $50 \mathrm{nM} \mathrm{SG}$. After incubation for $30 \mathrm{~min}$ at $37{ }^{\circ} \mathrm{C}$ in $5 \% \mathrm{CO}_{2}$ with or without different inhibitors, cells were stimulated with $100 \mathrm{nM}$ PMA, $250 \mathrm{ng} / \mathrm{ml}$ anti-Fas antibody or various concentrations of $\mathrm{H}_{2} \mathrm{O}_{2}$, after which the plates were sealed and loaded in a fluorescence Infinite M1000 plate reader (Tecan, Mechelen,
Belgium) and incubated at $37^{\circ} \mathrm{C}$. SG fluorescence was measured every $10 \mathrm{~min}$ for various periods. Cells treated with $0.2 \%$ Triton $\mathrm{X}-100$ were used as control and experiments were performed in triplicate.

\section{NADPH oxidase activity}

Superoxide production was measured as lucigenin-amplified chemiluminescence using a Biolumat 9505 apparatus (Berthold, Germany) as described [26]. After different treatments, PMN were diluted to $8 \times 10^{5} \mathrm{PMN}$ per $\mathrm{ml}$ in $250 \mu \mathrm{l}$ RPI medium supplemented with $0.5 \mathrm{mg} / \mathrm{ml}$ lucigenin. Then, $50 \mu \mathrm{l}$ PBS containing 600 nM PMA was added and the kinetics of superoxide production was measured at $37{ }^{\circ} \mathrm{C}$ for up to $60 \mathrm{~min}$. As the kinetics of activation was the same for all reaction mixtures, we used the heights of the peaks to express the results as percentages of control.

\section{Live cell imaging}

Neutrophils were diluted to $15 \times 10^{4} \mathrm{PMN}$ per $\mathrm{ml}$ in an eightchambered 1.0 borosilicate coverglass system (Nalge Nunc International, Rochester, NY, USA) coated with $0.1 \%$ poly-L-lysine and incubated for $30 \mathrm{~min}$ at room temperature (RT) in the presence of $3 \mu \mathrm{g} / \mathrm{ml}$ PI, 1:500 Annexin V-Alexa 488 solution (ANN), $10 \mu \mathrm{g} / \mathrm{ml}$ Hoechst 33342 solution (HB), $50 \mathrm{nM}$ TMRM, $1 \mu \mathrm{M}$ DCFDA or 50 nM SG. Cells were imaged using an Application Solution MultiDimensional Workstation (AS-MDW) equipped with a DM IRE2 microscope with a PIFOC P-Piezo element-driven HCX PL APO $63 \times / 1.3$, a $75-\mathrm{W}$ Xenon burner (with monochromator) set at $2 \mathrm{~mW}$ and a 12-bit CoolSNAP HQ camera (Leica Microsystems, Wetzlar, Germany). Cell morphology was observed by using differential interference contrast (DIC). Hoechst 33342 was excited at $380 \mathrm{~nm}$ and emission was detected using a BP340/80/FT400/LP425 filter cube. ANN, DCFDA and SG were excited at 490, 480 and $490 \mathrm{~nm}$, respectively, and emission was detected using a BP470/40/FT500/ BP525/50 filter cube. PI and TMRM were excited at 533 and $540 \mathrm{~nm}$, respectively, and emission was detected using a BP515560/FT580/LP590 filter cube. Phototoxicity and photobleaching were prevented by minimizing the exposure time for fluorescence excitation $(<100 \mathrm{~ms})$ and setting the camera at gain 2 and $2 \times 2$ binning. Cells were monitored for various times, and multiple image stacks were captured every minute, unless indicated otherwise. Different focal planes were set at $1-\mu \mathrm{m}$ intervals to prevent loss of focus of nonadherent cells. Poly-Lys treatment did not affect the kinetics and morphology of induced cell death of neutrophils.

From each image stack, maximum intensity projections (for PI, ANN, HB, TMRM, DCFDA, SG) and autofocus images (for DIC) were made for each time point by using a script developed in house for ImageJ $1.31 \mathrm{i}$ public domain imaging software. 3D deconvolution (iterative restoration based on calculated PSFs) was performed on Image sequences of PI, ANN, HB, TMRM, DCFDA and SG using the Volocity software 5.2.0 (PerkinElmer, Coventry, UK). Subsequent montages of the Multi-tiff time series and threechannel overlays were made in ImageJ 1.31i.

\section{Confocal microscopy}

Isolated human neutrophils were analyzed by confocal microscopy as previously described [50]. In brief, human neutrophils on uncoated glass coverslips were placed in 24-well plates for $30 \mathrm{~min}$ at RT. Cells were left unstimulated or were stimulated with 100 $\mathrm{nM}$ PMA for various periods up to $60 \mathrm{~min}$ at $37{ }^{\circ} \mathrm{C}$ in $5 \% \mathrm{CO}_{2}$. 
Subsequently, cells were fixed with 4\% paraformaldehyde for 15 $\mathrm{min}$ at RT. They were permeabilized with acetone at $-20{ }^{\circ} \mathrm{C}$ for 5 min. After rehydration with PBS at RT, cells were incubated with blocking buffer (PBS supplemented with $5 \mathrm{mg} / \mathrm{ml} \mathrm{BSA}+0.5 \mathrm{mg} / \mathrm{ml}$ $\mathrm{NaN}_{3}+10 \%$ FCS) overnight at $4{ }^{\circ} \mathrm{C}$. They were incubated with or without primary anti-LC3 antibody $(10 \mu \mathrm{g} / \mathrm{ml})$ for $1 \mathrm{~h}$ at RT. After 10 washing steps with PAB (PBS supplemented with $5 \mathrm{mg}$ / $\mathrm{ml} \mathrm{BSA}+0.5 \mathrm{mg} / \mathrm{ml} \mathrm{NaN}_{3}$ ), cells were incubated with 1:500 secondary goat anti-mouse antibody coupled to Alexa 488 for $1 \mathrm{~h}$ at RT, and DAPI was added at $10 \mu \mathrm{g} / \mathrm{ml}$ for another $10 \mathrm{~min}$. Then, cells were washed 10 times with PAB, followed by a final rinse with deionized water, after which they were mounted with gelvatol and sealed with nail polish. Confocal images were captured with a Leica Sp5 AOBS confocal microscope (Leica, Mannheim, Germany). Images were taken by using a 63× HCX PL Apo 1.4 oil objective. DAPI was excited with a UV diode laser at $405 \mathrm{~nm}$ and Alexa 488 by using the 488 line of a Multi Argon laser. Z-sections were made at the resolution limit, in this case $0.118 \mathrm{~nm}$, to produce a high-resolution stack suitable for 3D deconvolution and reconstruction. 3D iterative restoration based on measured PSFs and 3D reconstruction was performed using the Volocity software 5.2.0 (PerkinElmer). NET cell death features and kinetics were observed in the presence and the absence of fluorescent dyes. Consequently, the observed PMA-induced cell death was not due to phototoxicity.

\section{Electron microscopy}

Freshly purified neutrophils were allowed to adhere to glass coverslips in RPMI 1640 containing 4\% FCS. After incubation for $30 \mathrm{~min}$ at $37{ }^{\circ} \mathrm{C}$ in $5 \% \mathrm{CO}_{2}$, cells were stimulated with $100 \mathrm{nM}$ PMA or left untreated. Neutrophils were then fixed in $0.1 \mathrm{M} \mathrm{Na}$ cacodylate buffer containing $4 \%$ formaldehyde and $2.5 \%$ glutaraldehyde for $3 \mathrm{~h}$ at $4{ }^{\circ} \mathrm{C}$ and overnight in new fixative at $4{ }^{\circ} \mathrm{C}$. After washing with $0.1 \mathrm{M} \mathrm{Na}$ cacodylate buffer at $4{ }^{\circ} \mathrm{C}$, neutrophils were post-fixed in $1 \% \mathrm{OsO}_{4}$ with $1.5 \% \mathrm{~K}_{3} \mathrm{Fe}(\mathrm{CN})_{6}$ in $0.1 \mathrm{M} \mathrm{Na}$ cacodylate buffer ( $\mathrm{pH}$ 7.2). Samples were dehydrated through a graded ethanol series, and a bulk staining with $2 \%$ uranyl acetate was included at the $50 \%$ ethanol step. The coverslips were then embedded on molds containing pure Spurr's resin and left to solidify at $70{ }^{\circ} \mathrm{C}$. Ultrathin sections made with a Leica EM UC6 ultra microtome were post-stained in a Leica EM AC20 for $40 \mathrm{~min}$ in uranyl acetate at $20^{\circ} \mathrm{C}$ and for $10 \mathrm{~min}$ in lead stain at $20^{\circ} \mathrm{C}$. Grids were viewed with a 1010 transmission electron microscope (JEOL, Japan) operating at $80 \mathrm{kV}$.

\section{Western blotting}

Freshly isolated neutrophils $\left(5 \times 10^{5} \mathrm{PMN}\right.$ per $\left.\mathrm{ml}\right)$ from either healthy volunteers or CGD patients were pelleted and resuspended in lysis buffer containing 1\% Triton X-100, one tablet of Complete Mini protease inhibitor cocktail per $5 \mathrm{ml}$ lysis buffer and supplemented with $1 \mathrm{mM}$ PMSF and $1 \mathrm{mM}$ Pefabloc-SC. After incubating the lysate for $15 \mathrm{~min}$ on ice, cell debris was removed by centrifugation. Subsequently, $30 \mu \mathrm{g}$ proteins was separated in $8 \%$ SDS-PAGE. Western blots were analyzed by overnight incubation with 1:1 500 anti-gp91 ${ }^{\text {phox }}$ antibody and 1:6 000 anti-tubulin conjugated to HRP, followed by incubation with HRP-coupled secondary antibody at 1:7000.

\section{Statistics}

The results were analyzed with a two-way ANOVA and a
Bonferroni post-test (GraphPad Prism version 5, San Diego, CA, USA). Significance was accepted at $P<0.05$, unless indicated otherwise.

\section{Acknowledgments}

Special thanks for the enduring help with blood withdrawal go to Dr Eva De Wash and Els Bergé (KUL campus Kortrijk, Department of Medicine), Dienst Bloedafname of Dr Erik De Logi (University Hospital Ghent), Dr Henk Louagie (AZ St-Lucas, Ghent) and Dr Rudy Mortier (IDEWE, Ghent). We thank Gabriel Stelle (UBA, Buenos Aires) for the support. We thank Dr Chris Guerin (DMBR-VIB, Ghent) for critical reading and helpful suggestions and Dr Amin Bredan (DMBR-VIB, Ghent) for editing the article. This research has been supported by Flanders Institute for Biotechnology (VIB) and various grants. European grants: FP6 ApopTrain, MRTN-CT-035624; FP7 EC RTD Integrated Project, ApoSys, FP7-200767; Belgian grants: Interuniversity Attraction Poles, IAP 6/18; Flemish grants: Fonds Wetenschappelijke Onderzoek Vlaanderen, 3G.0218.06; Ghent University grants: 01GC0205 and GROUP-ID consortium from the UGent MRP initiative. QR and TVB hold a postdoc mandate position from the 'Fonds voor Wetenschappelijk Onderzoek'. PV is holder of a Methusalem grant (BOF09/01M00709) from the Flemish Government.

\section{References}

1 Luo HR, Loison F. Constitutive neutrophil apoptosis: mechanisms and regulation. Am J Hematol 2008; 83:288-295.

2 Maianski NA, Maianski AN, Kuijpers TW, Roos D. Apoptosis of neutrophils. Acta Haematol 2004; 111:56-66.

3 Brinkmann V, Reichard U, Goosmann C et al. Neutrophil extracellular traps kill bacteria. Science 2004; 303:1532-1535.

4 Steinberg BE, Grinstein S. Unconventional roles of the NADPH oxidase: signaling, ion homeostasis, and cell death. Sci STKE 2007; 2007:e11.

5 Babior BM. NADPH oxidase: an update. Blood 1999; 93:1464-1476.

6 Fuchs TA, Abed U, Goosmann C et al. Novel cell death program leads to neutrophil extracellular traps. J Cell Biol 2007; 176:231-241.

7 Wang Y, Li M, Stadler S et al. Histone hypercitrullination mediates chromatin decondensation and neutrophil extracellular trap formation. J Cell Biol 2009; 184:205-213.

8 van den Berg JM, van Koppen E, Ahlin A et al. Chronic granulomatous disease: the European experience. PLoS ONE 2009; 4:e5234.

9 Brinkmann V, Zychlinsky A. Beneficial suicide: why neutrophils die to make NETs. Nat Rev Microbiol 2007; 5:577-582.

10 Neeli I, Khan SN, Radic M. Histone deimination as a response to inflammatory stimuli in neutrophils. $J$ Immunol 2008; 180:1895-1902.

11 Parseghian MH, Luhrs KA. Beyond the walls of the nucleus: the role of histones in cellular signaling and innate immunity. Biochem Cell Biol 2006; 84:589-604.

12 Buchanan JT, Simpson AJ, Aziz RK et al. DNase expression allows the pathogen group A Streptococcus to escape killing in neutrophil extracellular traps. Curr Biol 2006; 16:396-400.

13 Beiter K, Wartha F, Albiger B, Normark S, Zychlinsky A, 
Henriques-Normark B. An endonuclease allows Streptococcus pneumoniae to escape from neutrophil extracellular traps. Curr Biol 2006; 16:401-407.

14 Clark SR, Ma AC, Tavener SA et al. Platelet TLR4 activates neutrophil extracellular traps to ensnare bacteria in septic blood. Nat Med 2007; 13:463-469.

15 Guimaraes-Costa AB, Nascimento MT, Froment GS et al. Leishmania amazonensis promastigotes induce and are killed by neutrophil extracellular traps. Proc Natl Acad Sci USA 2009; 106:6748-6753.

16 Kessenbrock K, Krumbholz M, Schonermarck U et al. Netting neutrophils in autoimmune small-vessel vasculitis. Nat Med 2009; 15:623-625.

17 Bruns S, Kniemeyer O, Hasenberg M et al. Production of extracellular traps against Aspergillus fumigatus in vitro and in infected lung tissue is dependent on invading neutrophils and influenced by hydrophobin RodA. PLoS Pathog 2010; 6:e1000873.

18 Bianchi M, Hakkim A, Brinkmann V et al. Restoration of NET formation by gene therapy in CGD controls aspergillosis. Blood 2009; 114:2619-2622.

19 Urban CF, Reichard U, Brinkmann V, Zychlinsky A. Neutrophil extracellular traps capture and kill Candida albicans yeast and hyphal forms. Cell Microbiol 2006; 8:668-676.

20 Hakkim A, Furnrohr BG, Amann K et al. Impairment of neutrophil extracellular trap degradation is associated with lupus nephritis. Proc Natl Acad Sci USA 2010; 107:9813-9818.

21 Yost CC, Cody MJ, Harris ES et al. Impaired neutrophil extracellular trap (NET) formation: a novel innate immune deficiency of human neonates. Blood 2009; 113:6419-6427.

22 Degterev A, Hitomi J, Germscheid M et al. Identification of RIP1 kinase as a specific cellular target of necrostatins. Nat Chem Biol 2008; 4:313-321.

23 van Raam BJ, Sluiter W, de Wit E, Roos D, Verhoeven AJ, Kuijpers TW. Mitochondrial membrane potential in human neutrophils is maintained by complex III activity in the absence of supercomplex organisation. PLOS ONE 2008; 3:e2013.

24 Borregaard N, Herlin T. Energy metabolism of human neutrophils during phagocytosis. J Clin Invest 1982; 70:550-557.

25 Maianski NA, Geissler J, Srinivasula SM, Alnemri ES, Roos D, Kuijpers TW. Functional characterization of mitochondria in neutrophils: a role restricted to apoptosis. Cell Death Differ 2004; 11:143-153.

26 Remijsen Q, Vanden Berghe T, Parthoens E, Asselbergh B, Vandenabeele P, Willems J. Inhibition of spontaneous neutrophil apoptosis by parabutoporin acts independently of NADPH oxidase inhibition but by lipid raft-dependent stimulation of Akt. J Leukoc Biol 2009; 85:497-507.

27 Kourtis N, Tavernarakis N. Autophagy and cell death in model organisms. Cell Death Differ 2009; 16:21-30.

28 Kroemer G, Galluzzi L, Vandenabeele P et al. Classification of cell death: recommendations of the Nomenclature Committee on Cell Death 2009. Cell Death Differ 2009; 16:3-11.

29 Kabeya Y, Mizushima N, Yamamoto A, Oshitani-Okamoto S, Ohsumi Y, Yoshimori T. LC3, GABARAP and GATE16 localize to autophagosomal membrane depending on form-II formation. J Cell Sci 2004; 117:2805-2812.

30 Yamamoto A, Tagawa Y, Yoshimori T, Moriyama Y, Masaki R,
Tashiro Y. Bafilomycin A1 prevents maturation of autophagic vacuoles by inhibiting fusion between autophagosomes and lysosomes in rat hepatoma cell line, H-4-II-E cells. Cell Struct Funct 1998; 23:33-42.

31 Eskelinen EL. New insights into the mechanisms of macroautophagy in mammalian cells. Int Rev Cell Mol Biol 2008; 266:207-247.

32 Levine B, Kroemer G. Autophagy in the pathogenesis of disease. Cell 2008; 132:27-42.

33 Yousefi S, Simon HU. Autophagy in cells of the blood. Biochim Biophys Acta 2009; 1793:1461-1464.

34 von Gunten S, Yousefi S, Seitz M et al. Siglec-9 transduces apoptotic and nonapoptotic death signals into neutrophils depending on the proinflammatory cytokine environment. Blood 2005; 106:1423-1431.

35 Beertsen W, Willenborg M, Everts V et al. Impaired phagosomal maturation in neutrophils leads to periodontitis in lysosomal-associated membrane protein-2 knockout mice. $J$ Immunol 2008; 180:475-482.

36 Huang J, Canadien V, Lam GY et al. Activation of antibacterial autophagy by NADPH oxidases. Proc Natl Acad Sci USA 2009; 106:6226-6231.

37 Mitroulis I, Kourtzelis I, Kambas K et al. Regulation of the autophagic machinery in human neutrophils. Eur J Immunol. 2010; 40:1461-1472.

38 Chen Y, Azad MB, Gibson SB. Superoxide is the major reactive oxygen species regulating autophagy. Cell Death Differ 2009; 16:1040-1052.

39 Fadeel B, Ahlin A, Henter JI, Orrenius S, Hampton MB. Involvement of caspases in neutrophil apoptosis: regulation by reactive oxygen species. Blood 1998; 92:4808-4818.

40 Hampton MB, Stamenkovic I, Winterbourn CC. Interaction with substrate sensitises caspase- 3 to inactivation by hydrogen peroxide. FEBS Lett 2002; 517:229-232.

41 Wilkie RP, Vissers MC, Dragunow M, Hampton MB. A functional NADPH oxidase prevents caspase involvement in the clearance of phagocytic neutrophils. Infect Immun 2007; 75:3256-3263.

42 Boya P, Gonzalez-Polo RA, Casares N et al. Inhibition of macroautophagy triggers apoptosis. Mol Cell Biol 2005; 25:1025-1040.

43 Eisenberg-Lerner A, Bialik S, Simon HU, Kimchi A. Life and death partners: apoptosis, autophagy and the cross-talk between them. Cell Death Differ 2009; 16:966-975.

44 Scherz-Shouval R, Shvets E, Fass E, Shorer H, Gil L, Elazar Z. Reactive oxygen species are essential for autophagy and specifically regulate the activity of Atg4. EMBO J 2007; 26:1749-1760.

45 Petiot A, Ogier-Denis E, Blommaart EF, Meijer AJ, Codogno P. Distinct classes of phosphatidylinositol 3'-kinases are involved in signaling pathways that control macroautophagy in HT-29 cells. J Biol Chem 2000; 275:992-998.

46 Sheppard FR, Kelher MR, Moore EE, McLaughlin NJ, Banerjee A, Silliman CC. Structural organization of the neutrophil NADPH oxidase: phosphorylation and translocation during priming and activation. J Leukoc Biol 2005; 78:1025-1042.

47 Remijsen Q, Vandenabeele P, Willems J, Kuijpers TW. Reconstitution of protection against Aspergillus infection in chronic granulomatous disease (CGD). Blood 2009; 114:3497. 
48 Yousefi S, Mihalache C, Kozlowski E, Schmid I, Simon HU. Viable neutrophils release mitochondrial DNA to form neutrophil extracellular traps. Cell Death Differ 2009; 16:14381444.

49 Vanden Berghe T, Kalai M, van Loo G, Declercq W, Vandena- beele P. Disruption of HSP90 function reverts tumor necrosis factor-induced necrosis to apoptosis. J Biol Chem 2003; 278:5622-5629.

50 Allen LA. Immunofluorescence and confocal microscopy of neutrophils. Methods Mol Biol 2007; 412:273-287.

(Supplementary information is linked to the online version of the paper on the Cell Research website.) 